\title{
Progress on the Development of the UAS C2 Link and Supporting Spectrum - from LOS to BLOS
}

\author{
Robert J. Kerczewski \\ NASA Glenn Research Center \\ 21000 Brookpark Rd \\ Cleveland, OH 44135 \\ +1216433 3434 \\ rkerczewski@nasa.gov
}

\author{
James H. Griner \\ NASA Glenn Research Center \\ 21000 Brookpark Rd \\ Cleveland, OH 44135 \\ +12164335787 \\ jgriner@nasa.gov
}

\author{
William D. Bishop \\ Jacobs Engineering \\ 21000 Brookpark Rd \\ Cleveland, OH 44135 \\ $+12164333473$ \\ william.d.bishop@nasa.gov
}

\author{
David W. Matolak \\ University of South Carolina \\ Columbia, SC 29208 \\ +1 803-777-8334 \\ matolak@cec.sc.edu
}

\author{
Jeffrey D. Wilson \\ NASA Glenn Research Center \\ 21000 Brookpark Rd \\ Cleveland, OH 44135 \\ $+12164333513$ \\ Jeffrey.d.wilson@nasa.gov
}

\begin{abstract}
In order to provide for the safe integration of unmanned aircraft systems (UAS) into the National Airspace System, the control and non-payload communications (CNPC) link connecting the ground-based pilot with the unmanned aircraft must be highly reliable and robust, based upon standards that enable certification. Both line-of-sight (LOS) links using terrestrial-based communications and beyond-lineof-sight (BLOS) links using satellite communications are required to support UAS operations. The development of standards has been undertaken by RTCA Special Committee 228 (SC-228), with supporting technical data developed by NASA under the UAS in the National Airspace (NAS) Project. As a result of this work minimum operational performance standards (MOPS) have been completed and published for the LOS CNPC system. The second phase of work, for both NASA and RTCA involves the BLOS CNPC systems. The development of technical data to support MOPS development for UAS BLOS satellite-based CNPC links has now been initiated by NASA, and RTCA SC-228 has organized itself to begin the MOPS development process. This paper will provide an overview of the work that has been completed to date by the Communications Subproject in support of LOS C2 communications for UAS followed by an update of plans and progress for the BLOS phase of the project, with the focus on the UAS C2 spectrum aspects.
\end{abstract}

\section{TABLE OF CONTENTS}

1. INTRODUCTION

2. Propagation STUdies: L-BAND AND C-

BAND.

3. CNPC RAdIO Flight TESTING..........................4

5. STUDIES FOR BLOS SPECTRUM..........................4

5. PHASE 2 - STUDIES FOR BLOS CNPC ...............7

6. CONCLUSION.............................................................8

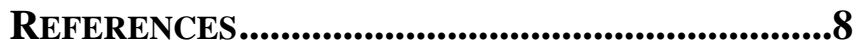

BIOGRAPHIES..........................................................9

\section{INTRODUCTION}

To enable unmanned aircraft system (UAS) operations to progress from occasional missions requiring specific authorization to routine access to the airspace supporting large numbers of UAS operating whenever and wherever needed, the development and validation of key systems supporting safe UAS and airspace operations is required. In particular, the command and control (C2) link must be highly reliable and robust. A specific requirement is that it must operate using aviation safety radiofrequency spectrum. Both line-of-sight (LOS) links using terrestrial-based communications and beyond-line-of-sight (BLOS) links using satellite communications must be developed. Performance standards must be developed and validated to enable UAS to be certified that they meet required performance levels.

NASA has been executing the UAS Integration in the National Airspace System (NAS) Project since 2012 to remove technical barriers to UAS integration in such areas as sense and avoid, separation assurance, human/systems integration and C2 communications. During the 2012 to 2016 phase of the Communication Subproject, the focus was on radio line-of-sight (LOS) C2 links using terrestrial-based communications. Prototype $\mathrm{C} 2$ radios were developed, bench-tested and flight tested to demonstrate data transfer performance, coverage distance and limits, signal loss and recovery and handoffs between ground stations in a variety of terrain types. C2 systems and networks modeling and simulation added to results that supported the development of minimum operational performance standards (MOPS) under RTCA Special Committee 228 (SC-228), and supported the allocation of spectrum for $\mathrm{C} 2$ at the international regulatory level. Propagation studies based on channel sounding campaigns were conducted to measure the characteristics of the air ground channel in L-Band and C-Band, supporting 
radio system design and modeling and spectrum compatibility analysis. Spectrum sharing studies and related analyses performed during this period supported the acquisition of new provisional frequency allocations for UAS C2 in Fixed Satellite Service (FSS) frequency bands.

In the next phase of the Communications Subproject, planned for 2017-2020, the focus will be beyond radio line-of-sight (BLOS) C2 links. Similar to the LOS Phase, the development, bench-testing and flight-testing of $\mathrm{C} 2$ radios for the satellite $\mathrm{C} 2$ link will be a key element. Using portions of FSS spectrum in $\mathrm{Ku}$ Band and $\mathrm{Ka}$ Band, performance validation and development of technical data to support MOPS development will be undertaken. Characterization of the air-space and ground-space channels will be performed. Importantly for the completion of the spectrum allocations, characterization of the interference environment between the UAS radio and terrestrial systems that operate in these bands will be performed - both terrestrial-to-UAS interference and UAS-to-terrestrial system interference will be studied.

This paper will provide an overview of the work that has been completed to date by the Communications Subproject in support of LOS C2 communications for UAS, followed by an update of plans and progress for the BLOS phase of the project, with the focus on the UAS $\mathrm{C} 2$ spectrum aspects.

\section{Propagation Studies: L-BAND AND C-BAND}

An important aspect of CNPC standards development involves understanding of the air-ground (AG) communication channel being used. Two frequency ranges considered likely to be applied to LOS UAS CNPC links are in L-Band (960-1164 MHz) and C-Band (5030-5091 MHz). NASA set out to study the AG channel in these bands by conducting a series of flight tests to measure the propagation characteristics of these channels and develop channel models to enable accurate analysis of radio performance [1].

Measurements of the CNPC AG channels were performed evaluated using a two-part measurement system consisting of a flight segment and a ground segment. Spread spectrum signals were transmitted from a self-contained, mobile ground platform equipped with radio frequency test electronics and a 60-foot tall extendable antenna mast. Test signals are received and recorded in-flight on-board a jet research aircraft equipped with specialized signal detection equipment. Propagation data was recorded while the aircraft executed a scripted set of flight maneuvers in airspace in the vicinity of the ground transmitter. Equipment in the air and ground segments was synchronized using Global Positioning System (GPS) timing to allow precise measurement of the desirable line-of-sight signal as well as the generally undesirable, multipath signals. The ground-mobile equipment is relocated to various terrestrial settings across the United States to quantify effects of local terrain and ground clutter on the AG channels.
A dual-band channel sounder is the principal equipment used to receive and record the line-of-sight and reflected (multipath) radio signals in the propagation tests. The custom channel sounder system consists of one transmit unit, two receive units, and attendant control and signal processing equipment. The sounder transmitter unit simultaneously produces signals in the L-Band (960-977 MHz) and C-band (5030-5091 MHz). Each of two identical sounder receiver (Rx) units receives simultaneously in both bands. This arrangement provides two physically separate receivers for each band (two L-band and two C-band) that can be connected to four separate antennas, i.e. a singleinput/multiple-output (SIMO) system for each band. The Lband signal bandwidth is approximately $5 \mathrm{MHz}$, and the $\mathrm{C}$ band signal bandwidth approximately $50 \mathrm{MHz}$.

The channel sounder system precisely records amplitude, phase and timing characteristics of the signals transmitted from the ground terminal and received on the aircraft. Processing of the data produces channel impulse responses, quantitative information on signals reflected by terrain and ground structures. The use of multiple aircraft antennae also allowed measurement of airframe shadowing effects of aircraft wings, engines, or other protuberances [2].

As the AG channel can vary significantly across different types of terrain, several terrain types were studied: mountainous, hilly, flat terrain, near-urban and suburban and over salt and fresh water. Figure 1 shows various locations in the United States where flight tests were performed to gather AG channel data.

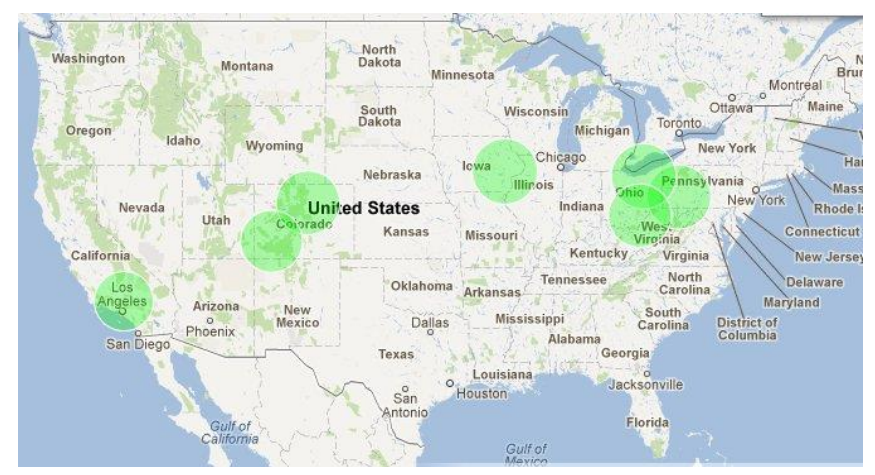

Figure 1 - AG channel measurement flight test locations.

The flight platform used in the propagation measurements is a NASA Glenn Research Center Lockheed model S-3B Orion jet aircraft. For the propagation testing, the baseline flight patterns are executed at 164-261 mph airspeed at altitudes of 480-1900 m above ground level. Two L-band and two Cband antennas are mounted on the underside surface of the aircraft. Figure 2 shows the aircraft and location of antennas. 


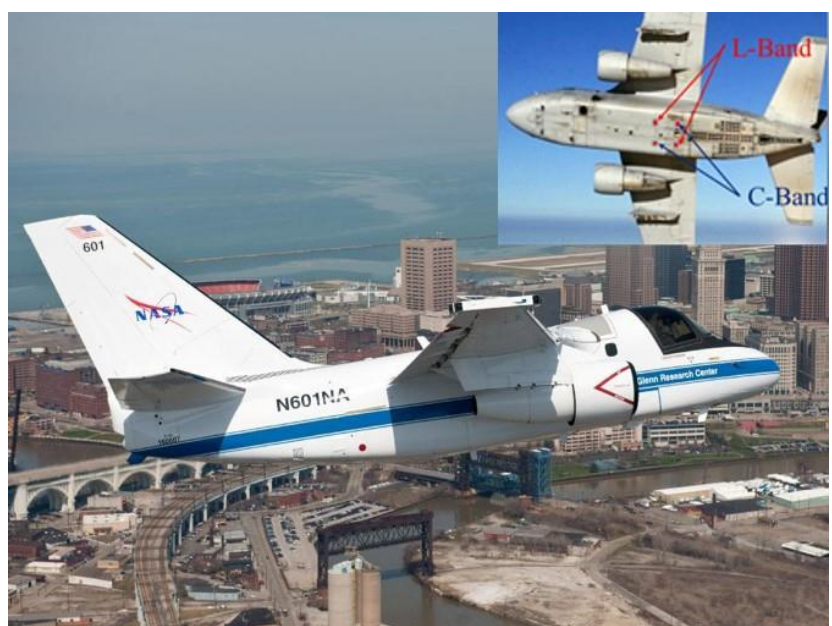

Figure 2 - Lockheed Orion S-3B research aircraft. Inset: antenna locations

Flight tests attempted to provide a complete set of $\mathrm{AG}$ channel characteristics by flying in directions parallel and perpendicular to the line from the aircraft to the ground transmitter location. Figure 3 provides an example for saltwater measurements near Oxnard, California [3, 4].

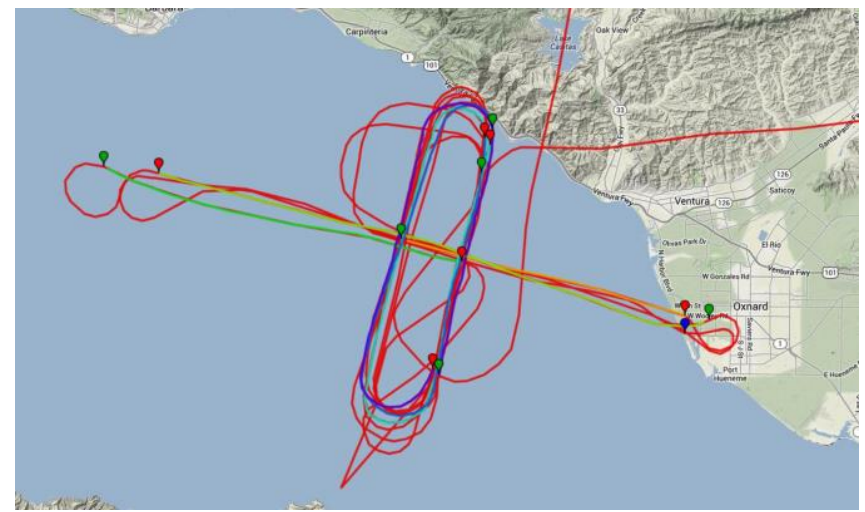

Figure 3 - Recorded Flight Tracks for Over Sea Propagation Measurements near Oxnard, California.

Several months of flight testing produced a large volume of channel impulse response data. The final NASA report on AG channel characterization results is being completed. Here we provide an examples for the over sea (salt water) channel.

For one of the flight tracks in Figure 3, measured and analytical path loss vs. link distance, for the two C-band receivers ( $\mathrm{Rx} 1$ and $\mathrm{Rx} 2$, in (a)), and the two L-band receivers (b) is plotted in Figure 4. The measured path loss for both bands is close to that of the free-space value, but slightly larger for short distances, and slightly less in C-band for large distances, likely attributable to aircraft antenna pattern effects. Some of the "lobing" structure of the theoretical tworay model can be seen in the measured data at the larger values of distance, and this is clearest for L-band. The curved-earth two ray model (including spherical earth divergence and sea-surface roughness effects) fits the measured path loss data better than the flat-earth two-ray and free space models, although the free space model predicts the mean value well for these link distances.

Path loss models and small scale fading parameters have been derived for the terrain types studied. Air frame shadowing models have been developed that, although specific to the aircraft used in the AG channel measurement campaign, nevertheless provide significant insight into shadowing effects.

The final aspect of the L-Band and C-Band propagation studies will be to prepare propagation data and channel models suitable for inclusion in the propagation databanks and propagation recommendations documents of the International Telecommunications Union. This will enable the use of these results in future studies performed under the ITU's auspices for international spectrum and regulatory activities and link design relevant to aeronautical radiocommunications.

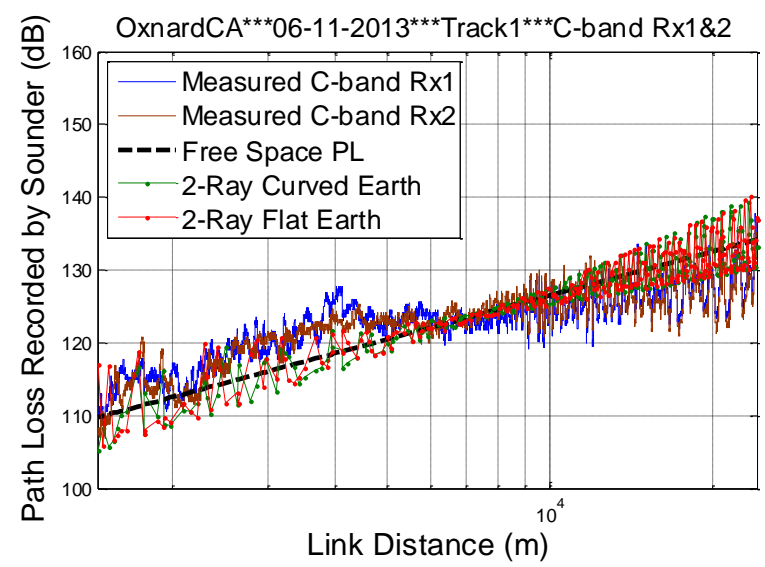

(a)

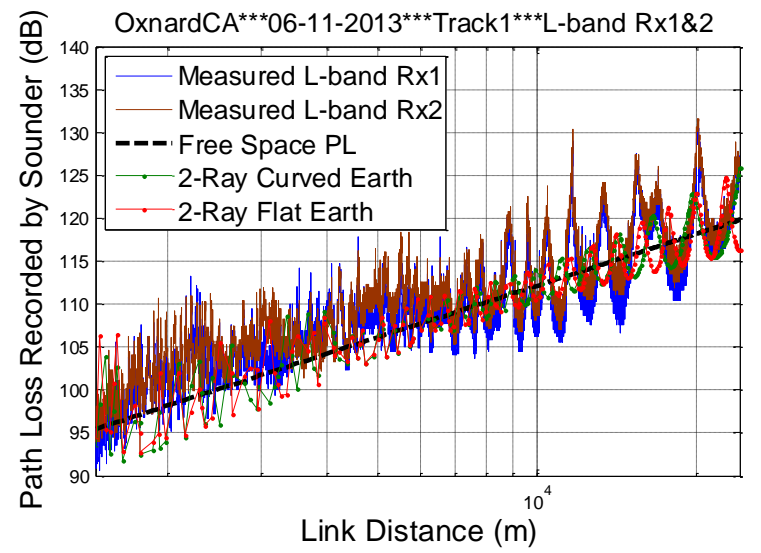

(b)

Figure 4. Measured results of $\mathrm{C}$-band path loss vs. distance for all receivers, Flight Track 1: (a) C-band, (b) L-band [4]. 


\section{CNPC Radio Flight Testing}

To develop the technical data required to support RTCA SC28, NASA's Glenn Research Center developed several iterations of a prototype CNPC radio, through a cooperative agreement with Rockwell Collins, Inc. Development steps undertaken to realize the prototype UAS CNPC system included investigation of signal waveforms and access techniques, development of representative CNPC radio hardware, and execution of relevant testing and validation activities. The purpose was not to manufacture the CNPC radio end product but rather to enable the study, demonstration, and validation of a typical CNPC system that allows safe and efficient communications within the L-band and C-band spectrum allocations. Prototype testing also allowed the team to and develop the necessary data to inform the requirements and standards development processes.

Flight test campaigns were conducted for five generations of radios. The generations progressed from an initial L-Band radio, through dual L-Band/C-Band radios, multi-ground station performance capability, and refinement of network, waveform and network connectivity performance.

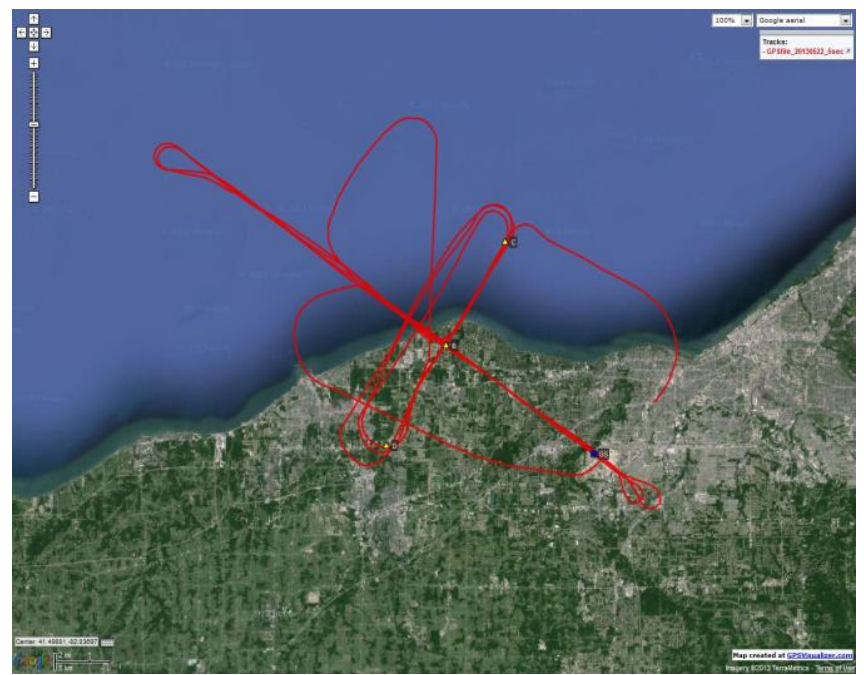

Figure 5. Flight Test \#1 Flight Track, 22 May 2013 Cleveland, Ohio.

The radios were installed on the NASA Glenn Research Center Lockheed model S-3B Orion aircraft for the flight tests. Flight tests consisted of multiple, pre-planned aircraft maneuvers and flight path segments. The objective of the flight test campaign was to operate the radios in an air-ground flight environment to determine possible limitations to communications range and data throughput performance, assess handoff performance between ground stations, and fully examine data transmission performance, in particular as it relates to requirements for control of UAS.

Typical flight tracks are shown in Figure 5. Example results of the flight test are plotted in Figure 6. A time scale is plotted along the horizontal axis (abscissa) of the figure, which encompasses the entire test activity from pre-flight ground and taxi operations through flight maneuvers. Signal strength is plotted along the vertical axis (Ordinate) in the top plot. Both the ground radio and aircraft radio data are presented on the same grid in red and blue traces, respectively. The calculated expected theoretical free space received signal strength is also show as the black line in this plot [5].

In graphs below the received signal strength trace, Figure 6 presents example data on average percentage frame loss at the aircraft and at the ground station receivers. When the CNPC communications path is transferring all data without error, the data is presented as $0 \%$ loss and no colored trace is visible on the grid. When errors occur in the radio link, the lost frame data creates a visible trace ranging from $1 \%$ up to $100 \%$ (total loss of radio link). Some frame loss was recorded during the aircraft course reversals, as expected.

The bottom three plots in Figure 6 show the range between the aircraft and ground station, the aircraft altitude and the aircraft roll angle. Range and aircraft maneuvers can be seen to correlate with changes in signal strength.

The second generation radio flight testing of the second generation CNPC radio added investigation of the performance of the radios in communicating with two independent ground stations separated by a distance nearly twice the design range of the radios. In particular, the handoff of the CNPC data communication between ground stations was examined [6]. Figure 7 shows the test system employed for the multi-ground station testing, and Figure 8 shows the ground station locations.

The testing demonstrated smooth transitions between ground stations. The networking tests showed that the median round trip propagation time between the airborne and ground radios was $225 \mathrm{~ms}$, including network and equipment processing times. The observed round trip times had a significant amount of variation, with the majority of samples between 171 and $267 \mathrm{~ms}$. This spread of $96 \mathrm{~ms}$ was consistent with the $10 \mathrm{~Hz}$ (100 ms) waveform configuration used for the tests. Thus, the maximum targeted $20 \mathrm{~Hz}(50 \mathrm{~ms})$ configuration should yield the least amount of delay variation. Traversal over the terrestrial Internet added an additional $55 \mathrm{~ms}$ round-trip for the location in Albany, Ohio. This brought the total round trip delay from the home network location to the aircraft via the ground station to over 0.25 seconds in the average case. Network hand-offs were shown to be seamless and transparent to the end user.

Flight testing continued for the subsequent radio generations as interaction with RTCA SC-228 provided refinements in CNPC radio requirements. Reports on the results of these tests are nearing completion.

\section{STUDIES FOR BLOS SPECTRUM}

During the time when the UAS LOS CNPC MOPS were being developed, activities required for the second phase of the UAS in the NAS Project and RTCA CNPC MOPS development were proceeding. The second phase focuses on BLOS CNPC using satellite communications. 


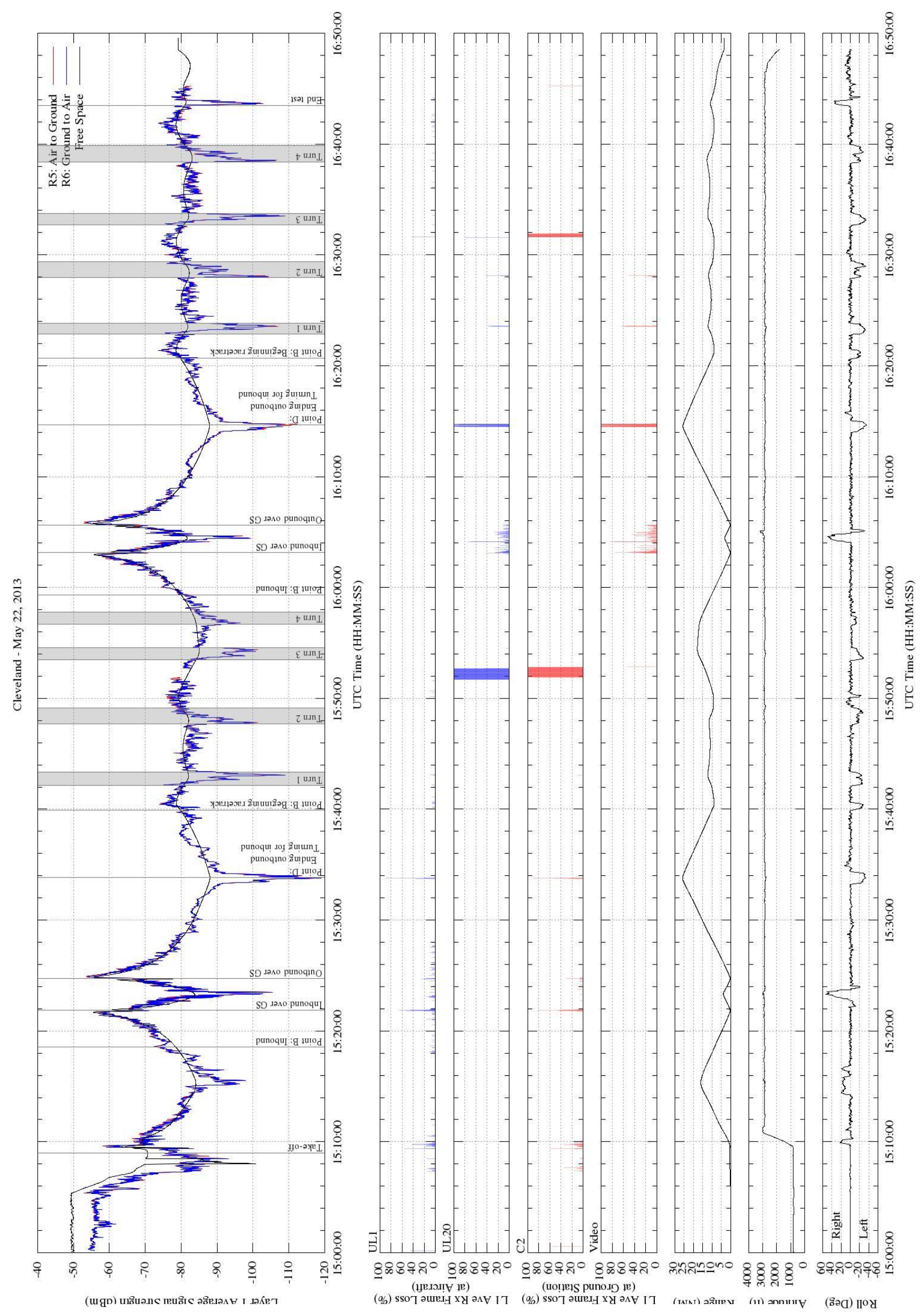

Figure 6. Flight Test 1 Radio Performance Data and Associated Aircraft ГГ4] 


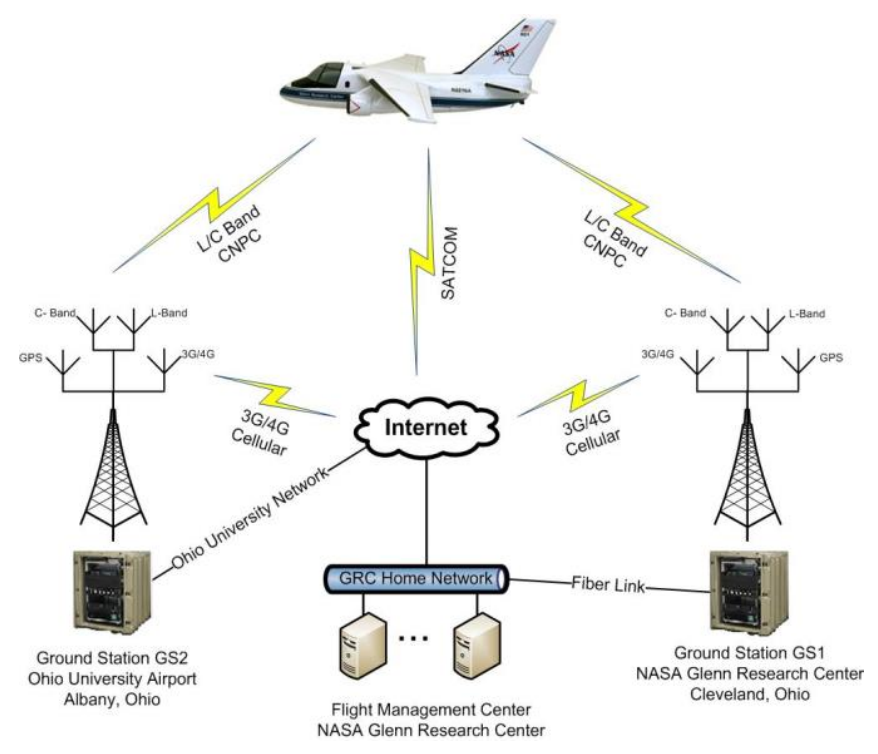

Figure 7. Multi-ground station test system.

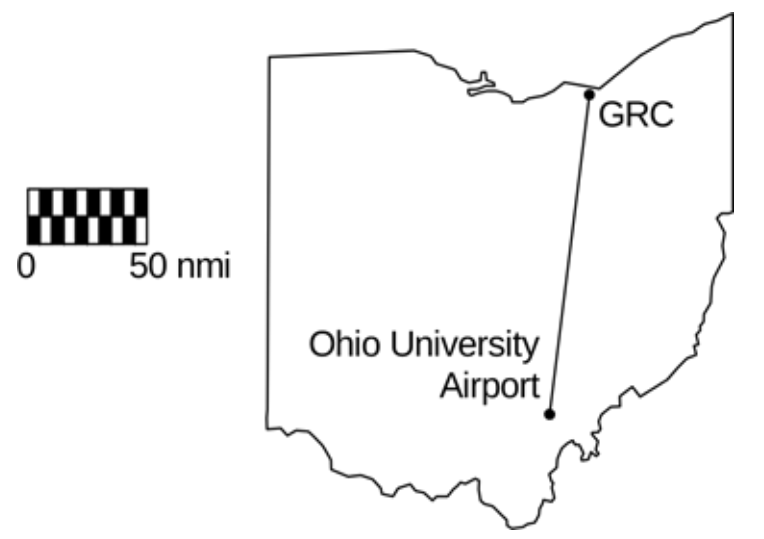

Figure 8. Locations of ground stations within Ohio at a separation distance of $133 \mathrm{nmi}$.

Spectrum requirements to support BLOS CNPC were addressed during the 2015 World Radiocommunication Conference (WRC-15). Existing spectrum allocations did not satisfy requirements for providing UAS BLOS CNPC. WRC-15 Agenda Item 1.5 considered "the use of frequency bands allocated to the fixed-satellite service not subject to Appendices 30, 30A and 30B for the control and non-payload communications of unmanned aircraft systems (UAS) in nonsegregated airspaces...", recognizing that existing satellite networks operating in the fixed satellite service (FSS) in the frequency bands at $14 / 12 \mathrm{GHz}$ (Ku-band) and $30 / 20 \mathrm{GHz}$ (Ka-band) have potential spectrum capacity to meet the requirements for UAS BLOS C2.

Agenda Item 1.5 required study of the impact of UAS CNPC operations on other services having allocations in the bands being proposed in FSS for UAS use. These sharing studies were undertaken during the first phase of NASA's UAS in the NAS. The studies and outcomes have been reported in $[7,8]$ and are briefly reviewed here.
Figure 9 shows the sharing study scenario. The Fixed Service (FS), which is terrestrial only, shares a primary spectrum allocation with the FSS in portions of both $\mathrm{Ku}-\mathrm{Band}$ and $\mathrm{Ka}-$ Band. Fixed FSS ground station locations can be coordinated so as not to exceed interference protection criteria protecting the FS from harmful interference. The interference case because complex when the FSS ground stations are mounted on UAS and are in motion.

Protection criteria are stated in both long-term and short term parameters. For Ku-Band, the long term protection criterion requires that the interference to noise power density, $\mathrm{I} / \mathrm{N}$, to the FS receiver shall not exceed $-10 \mathrm{~dB}$ for more than $20 \%$ of the year while the short term protection criteria requires that the $\mathrm{I} / \mathrm{N}$ shall not exceed $+20 \mathrm{~dB}$ for more than $1 \times 10-4 \%$ of

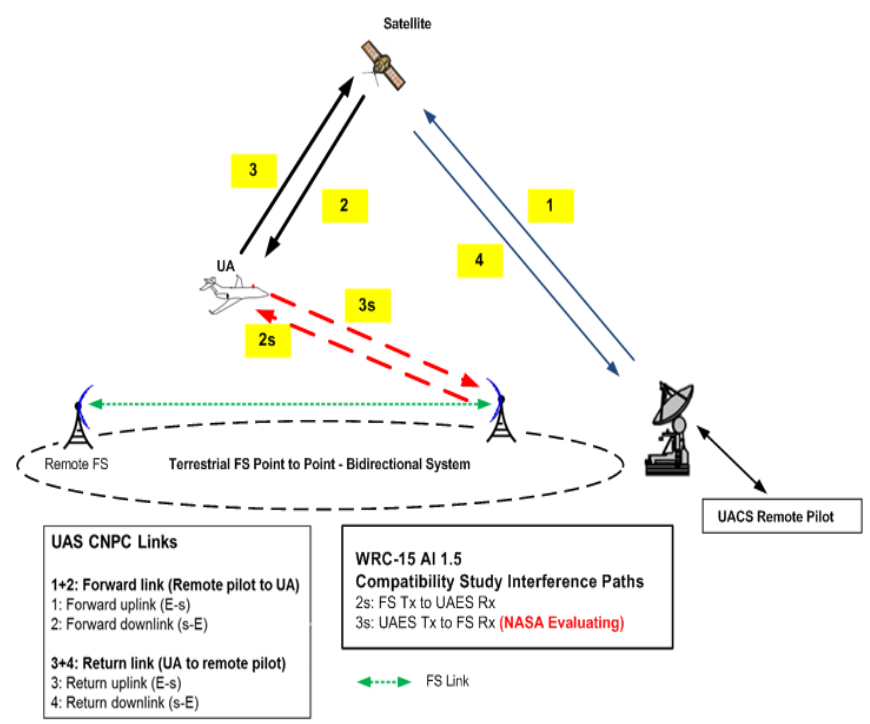

Figure 9. Sharing scenario for UAS BLOS CNPC in FSS bands.

the time. For Ka-Band, the protection criterion for the long term analysis is the same as that for Ku-band, while the shortterm protection criterion is that $\mathrm{I} / \mathrm{N}$ should not exceed $+14 \mathrm{~dB}$ for more than $0.01 \%$ of the time in any month and should not exceed $+18 \mathrm{~dB}$ for more than $0.0003 \%$ of the time in any month.

Because of the expected density of UAS in the airspace, the long-term protection criteria is met; there are not enough UAS within line-of-sight of a given FS receiver to exceed $20 \%$ of the time. The short-term protection criteria is harder to meet, since an exceedance of the threshold for as little as 3 seconds in a month will violate the criteria.

Figure 10 shows a cumulative distribution function (cdf) for long-term interference at Ku-Band, compared to the protection criterion (shown by the red diamond), derived through simulation. There is approximately $20 \mathrm{~dB}$ or more of margin. Figure 11 shows a cdf for the Ka-Band case, with an even larger margin. As long as the density of UAS does not increase significantly above expected numbers, long-term criteria will always be met. 
For the short-term criterion, the protection criterion margin is small for the Ku-Band case, shown in Figure 12. This margin disappears when higher antenna gains for FS receivers are considered, such that some constraints will be placed on UAS operations, particularly at high latitudes where the UAS antenna must operate at a low elevation angle.

WRC-15 adopted Resolution 155 to address Agenda Item 1.5. Resolution 155 specifies an allocation in Ka-Band covering only the $29.5-30.0 \mathrm{GHz}$ band for the uplink, effectively eliminating sharing difficulties since there are no FS allocations in that band segment. For Ku Band, the uplink allocation covered $14.0-14.47 \mathrm{GHz}$, but the issue of potential interference still needs to be addressed. The Resolution specifies a requirement for UAS earth stations to meet a power flux density (pfd) limit in order to protect the FS from interference. This specific pfd limit was not agreed to at WRC-15 and so must be further studied in order to develop a consensus at the next WRC in 2019.

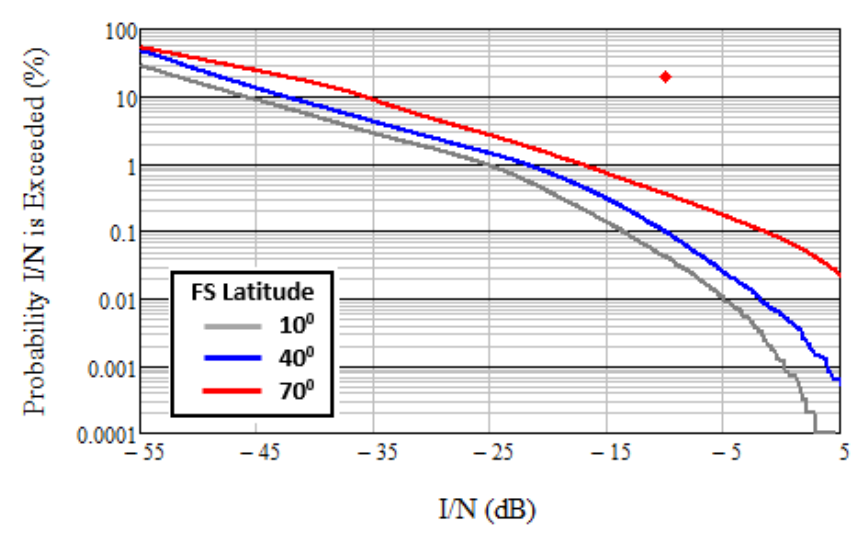

Figure 10. Long-term analysis results for $14.4 \mathrm{GHz}$ with FS station at $10^{\circ}, 4^{\circ}$, and $70^{\circ}$ latitude, $\mathrm{UA}$ at $3000 \mathrm{ft}$ above ground level, large $U A$ antenna.

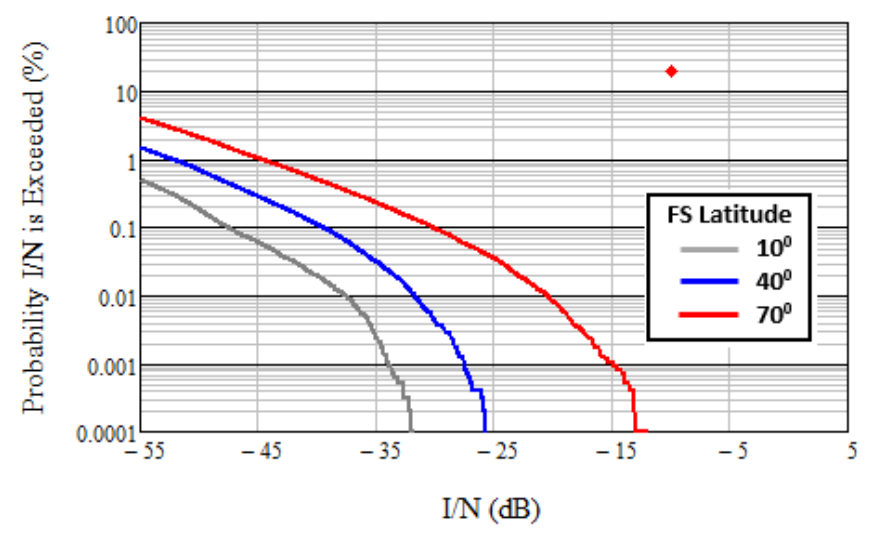

Figure 11. Long-term analysis results for $28.5 \mathrm{GHz}$ with FS station at $10^{\circ}, 4^{\circ}$, and $70^{\circ}$ latitude, $\mathrm{UA}$ at $3000 \mathrm{ft}$ above ground level, small $U A$ antenna.

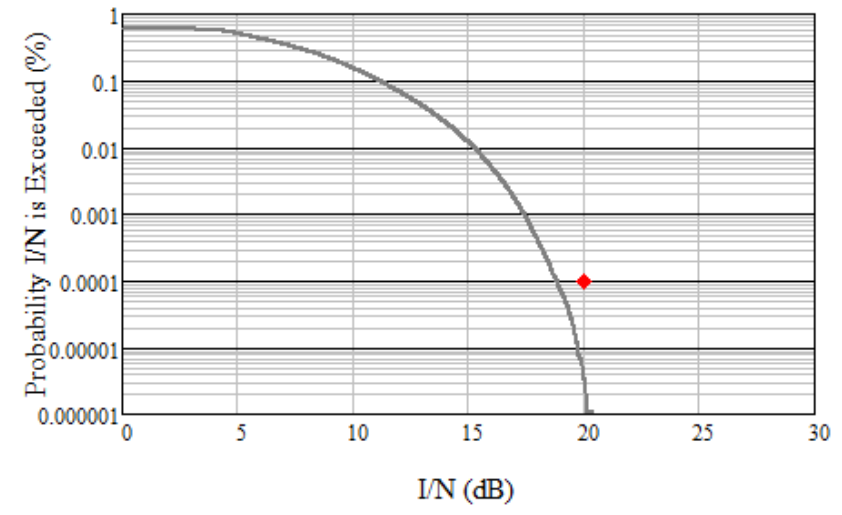

Figure 12. Short-term analysis for $14.4 \mathrm{GHz}$ with FS station at $70^{\circ}$ latitude, $\mathrm{UA}$ at $3000 \mathrm{ft}$ above ground level, large $\mathrm{UA}$ antenna.

\section{Phase 2 - STUdies For BLOS CNPC}

In the next phase of the UAS in the NAS Communications Subproject, planned for 2017-2020, attention will turn to BLOS CNPC links using satellite communications. The objective is again to provide the technical foundation for the development of standards, and to address other critical issues such as spectrum.

A development of prototype CNPC systems will be undertaken to enable bench-testing and flight-testing of CNPC radios for the BLOS CNPC link. It is anticipated that both Ku-Band and Ka-Band BLOS radios will be included in the test program to perform system validation and development of technical data to support MOPS development.

Characterization of the air-space and ground-space channel will be performed as a byproduct of prototype radio testing, although a dedicated propagation measurement campaign similar to that performed for the AG channel is not anticipated.

The allocation of spectrum at Ku-Band and Ka-Band for the CNPC application has progressed but is not complete. A particular problem exists for the Ku-Band allocation in terms of spectrum sharing with the FS. WRC-19 will consider the power flux density (pfd) limit that must be applied to UAS operations in order to prevent interference into the FS receivers. The pfd limit must be carefully considered because it will constrain BLOS CNPC operations.

Among the problems that must be considered in Phase 2 are realistic assessments of pfd levels resulting from CNPC UAS transmissions. Current study results have been analytic in nature but measured results are needed to validate the models employed in the analyses. For example, measured antenna performance, in situ (on-board the aircraft) and in flight is needed to improve the analysis. The effect of fuselage attenuation of received pfd on the ground is also of great interest. 
Approaches to mitigating the constraints imposed by pfd limits, such as spectral spreading, must also be investigating to ensure that pfd limits that will be agreed to at WRC-19 can be met while operating a practically implementable BLOS CNPC link.

WRC-15 Resolution 155 also requires that UAS BLOS CNPC receivers must accept interference from co-primary in-band services such as the FS. The aggregate effect of many FS transmitters, while constrained to operate within their required limits, will have the potential to impact the performance of the BLOS CNPC link. This potential problem must also be investigated and mitigation approaches developed and validated if needed.

\section{Conclusion}

Considerable progress has been made with regards to developing CNPC solutions for UAS systems operating in the National Airspace. An extensive propagation measurement campaign has been conducted by NASA for the air-ground channel in both L-Band and C-Band, covering a range of terrain types. Propagation statistics have been derived and detailed channel models have been developed.

Several generations of prototype CNPC radios for the LOS link have been developed and extensively flight-tested by NASA. Test results have been provided to the standards development process, resulting in refinements of the prototype CNPC radio and further testing and validation. As a result of this work, RTCA SC-228 has completed development of the LOS terrestrial CNPC system MOPS.

WRC-15 has approved Resolution 155, providing spectrum allocations for UAS BLOS CNPC, allowing testing and development of satellite communications-based CNPC systems to proceed.

NASA and RTCA SC-228 are now entering a second phase of CNPC development, testing and standards development. Testing of prototype BLOS CNPC radios will be undertaken by NASA to provide technical foundation for the development of the UAS CNPC satellite communications MOPS. Additional testing and analysis will support completion of the spectrum allocation process.

This work represents an aggressive effort to enable the integration of UAS into the national airspace within a relatively short period of time. The impact of this work will begin to be seen in the near future. The desire to apply UAS to a wide variety of purposes continues to increase.

\section{REFERENCES}

[1] ICAO Aeronautical Communications Panel, Working Group \#26, Information Paper 8, "L-Band and C-Band AirGround Channel Measurement Campaign”, March 2012.

[2] ICAO Aeronautical Communications Panel, Working Group \#28, Information Paper 3, "Update on L-Band and C-Band Air-Ground Channel Measurement Campaign", March 2013.

[3] ICAO Aeronautical Communications Panel, Working Group \#30, Information Paper 8, "L-Band and C-Band AirGround Channel Measurement \& Modeling for Over-Sea Conditions", March 2014.

[4] D. W. Matolak, R. Sun, "AG Channel Measurement \& Modeling Results for Over-Sea Conditions," (Report \#6) NASA Grant \#NNX12AR56G, 3 December 2013.

[5] ICAO Aeronautical Communications Panel, Working Group \#29, Information Paper 6, "Flight Tests of First Generation Prototype CNPC Radio”, September 2013.

[6] ICAO Aeronautical Communications Panel, Working Group \#32, Information Paper 3, "Ground Station Handoff Tests of a Prototype CNPC Radio", March 2014.

[7] Kerczewski, R. J., Wilson, J. D., and Bishop, W. D., "UAS CNPC Satellite Link Performance - Sharing Spectrum with Terrestrial Systems", 2016 IEEE Aerospace Conference, March 2016.

[8] Kerczewski, R. J , Wilson, J. D., and Bishop, W. D., "Satellite Communications for Unmanned Aircraft C2 Links - C-Band, Ku-Band and Ka-Band", $222^{\text {nd }} \mathrm{Ka}$ and Broadband Communications Conference, October 2016. 


\section{BIOGRAPHIES}

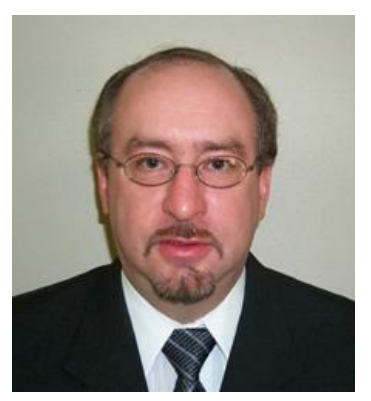

Robert J. Kerczewski has been involved with research and development of satellite and aeronautical communications systems and applications for the Analex Corporation (1982-1986) and NASA (1986-present). He holds a BEE degree from Cleveland State University (1982) and an MSEE degree from Case Western Reserve University (1987). He is currently the Spectrum Element Manager for the NASA's Unmanned Aircraft Systems Integration in the National Airspace System (UAS in the NAS) Communications Sub Project.

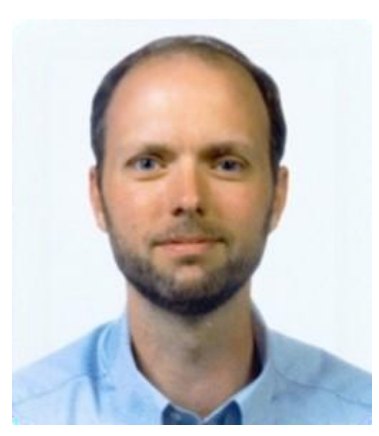

Jim Griner is a senior electrical engineer at the NASA Glenn Research Center, in Cleveland, $\mathrm{OH}$. He has over twenty years experience in computer network and system engineering for satellite and terrestrial communications, including extensive ground and flight testing. Currently, Mr. Griner is the project engineer for the communication portion of NASA's Unmanned Aircraft Systems integration in the NAS project. Mr. Griner has a BEE from Georgia Tech and MSEE from Case Western.

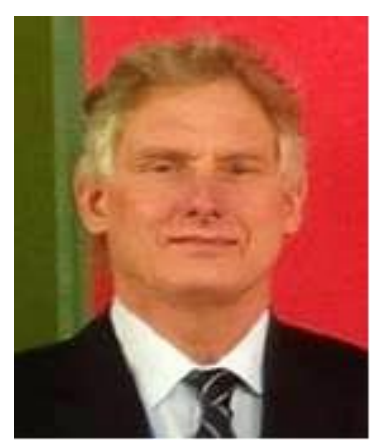

William D. Bishop is currently involved with the research, design and development of Unmanned Aircraft Systems Control Non-Payload Communications Systems at NASA in the Datalink and Spectrum Planning areas (2010present). He also has extensive and comprehensive wireless industryexperience commencing in 1986 in the RF design, planning, performance optimization and traffic management of wireless networks supporting all U.S. carriers in all deployed voice and data technologies, including 4G. His early efforts include the design, development and practical use of the Dual-Cell Concept (FOA - GTE Mobilnet, 1987), which increased offered load capacity in congested areas within a traditional reuse plan. He holds a BEE degree from Cleveland State University Fenn College of Engineering (1998), as well as an FCC General Radiotelephone License (1986).
David W. Matolak has over 20 years of experience in wireless communication system design, analysis, testing, and deployment, for a variety of applications. He received his B.S., M.S., and Ph.D. degrees from Penn State University, The University of Massachusetts, and The University of Virginia, respectively, all in electrical engineering. He worked with the Rural Electrification Administration, several companies (AT\&T Bell Laboratories, Lockheed-Martin, and MITRE) before joining academia at Ohio University. Since 2012 he has been with the University of South Carolina. Throughout his career he has worked on various types of communication systems, including terrestrial, satellite, and aeronautical, primarily at the physical and data link layers. His work has involved analyses, computer simulations, and experiments. He has been a visiting professor at the National Institute of Standards \& Technology, Boulder, $\mathrm{CO}$, the University of Malaga, Spain, and NASA Glenn Research Center. He is a member of Eta Kappa Nu, Sigma Xi, AAAS, AIAA, ASEE, URSI, and a senior member of IEEE.

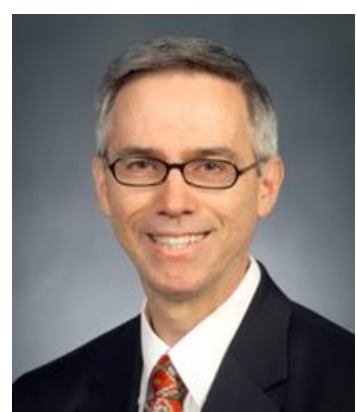

Jeffrey D. Wilson received the B.S. degree in physics magna cum laude from Bowling Green State University in 1976, and the M.S. and Ph.D. degrees in physics from the University of Illinois at Urbana-Champaign in 1978 and 1983, respectively. Since 1983, Dr. Wilson has been employed at NASA Glenn Research Center, Cleveland, Ohio. He spent the 1984-1985 academic year in postdoctoral study with the Air Force Thermionic Electronics Research (AFTER) Program at the University of Utah. His research efforts have focused on computational techniques to enhance the power, efficiency, and performance of coupledcavity, helical, and terahertz wave traveling-wave tubes (TWT's), the electromagnetic properties of metamaterials, quantum communications, and interference issues in $R F$ communications systems. Dr. Wilson is a Senior Member of IEEE. 